\title{
Severe Acute Epididymo-Orchitis Complicated with Abscess and Testicular Necrosis - Case Report
}

\section{(ㅇ)(우)(8)}

\section{Introduction}

Acute epididymo-orchitis (the inflammation of one or both testes and epididymis) is a common urological diagnosis encountered by urologists and by emergency and primary care physicians. It should be differentiated from testicular torsion - a true urological emergency. Furthermore, acute epididymitis can be complicated by testicular abscesses or by testicular infarction, due to spermatic cord swelling and by the impairment of blood flow - conditions that should be easily recognized and properly treated.

The diagnosis is usually established based on clinical symptoms and physical examination, but also further examinations could be helpful in confirming the epididymo-orchitis diagnosis and in identifying complications. Differentiation between epididymo-orchitis and testicular torsion can sometimes be difficult. However, a referral to the urologist when testicular torsion is suspected should not be delayed by an ultrasonographic (US) examination. Contrast-enhanced ultrasonography (CEUS), as well as color Doppler ultrasound (CDUS) and power Doppler ultrasound (PDUS), could be really useful in assessing the blood flow to the scrotum and the testes. Unlike CDUS and PDUS, CEUS could help in evaluating focal testicular complications of epididymitis and in establishing the extent of the unviable tissue (Lung PF et al. AJR Am J Roentgenol 2012;199: W345).

The aim of this case report is to demonstrate the clinical value of CEUS in the acute setting of testicular diseases.

\section{Case Report}

A 47-year-old patient presented to the urology department with edema and moderate swelling of the left side of the scrotum, accompanied by dysuria. The onset of the disease was sudden, one month ago, with intense nocturnal pain in the left testicle that lasted about $6 \mathrm{~h}$ accompanied by the swelling of the left scrotum. The patient was admitted to another medical care unit where a diagnosis of acute epididymo-orchitis was established, and a wide spectrum of antibiotics and analgesic therapy was initiated with the relief of pain. He was discharged after a few days and he was advised to continue the antibiotic therapy (ciprofloxacin) at home for one month. Given that after one month of treatment the swelling of the scrotum did not improve and the patient developed some episodes of low-grade fever (up to $37.8^{\circ} \mathrm{C}$ ), he was referred to our urology department.

The physical examination revealed swelling and erythema of the left scrotum. The left testicle was swollen, firm, and mildly tender to palpation. No other pathological findings were identified during the complete physical examination. Blood test results were within reference ranges. Urine culture showed a multi-drug-resistant, extended-spectrum, beta-lactamase-producing Klebsiella spp. urinary tract infection.

Abdominal ultrasonography was performed with no peculiar pathological findings. At the ultrasonographic examination of the scrotum using a GE Logiq 9 ultrasound machine and a linear probe $9 \mathrm{~L}$ (2.58 ), the left testicle appeared significantly diminished in size (with a volume of about $8 \mathrm{~cm}^{3}$ ) with considerable structural changes with hypo- and hyperechoic areas. The scrotal layers were significantly thickened with lymphedema, ( $\triangleright$ Fig. 1a). The Doppler ultrasound examination revealed marked epididymal congestion and only a few vascularized parenchymal areas of the testicle ( Fig. 1b). Ultrasound elastography showed a moderate increase of the testis stiffness, with an acoustic radiation force impulse (ARFI) of about $1.7 \mathrm{~m} / \mathrm{s}$. Contrast-enhanced harmonic ultrasonography was performed after intravenous contrast injection of SonoVue $(1.6 \mathrm{ml}$ with flush of $10 \mathrm{ml}$ saline solution), highlighting the lack of enhancement of the left testicle ( $\triangleright$ Fig. 2 ), which is suggestive of testicular ischemia. The right testicle was ultrasono- graphically normal.

Given the symptomatology and the extent of the testicular damage (almost the entire parenchyma), a left orchiectomy was performed. Intraoperatively, the surgeon identified the testicle with significant destruction of its structure. Areas of necrosis were observed on the surface and on a cut section ( $\triangleright$ Fig. $\mathbf{3}$ ). The resection specimen was sent to the pathology department. The histopathological examination revealed severe acute epididymo-orchitis with intratesticular abscess and destruction of $90 \%$ of the testicular parenchyma ( $\vee$ Fig. 4 ). The final diagnosis was a severe acute epididymo-orchitis complicated by abscess and necrosis.

\section{Discussion}

The symptomatology and clinical appearance of acute and non-acute scrotal diseases are commonly similar, with pain and swelling of the scrotum being the most frequent reasons for patients to seek medical care. Acute testicular diseases require fast diagnosis and treatment in order to preserve the viability of the tissue. Hemodynamically stable patients can be quickly examined by CEUS, without losing any precious time, in order to obtain valuable information. CEUS examination lasts less than $10 \mathrm{~min}$, thus making it suitable in emergency situations (Cokkinos DD et al. Insights Imaging 2013;4:185), especially as it can be available bedside.

Epididymo-orchitis is the most common cause of acute scrotum in men over 18 years old. Even if it is considered an infectious process, urine cultures are often negative (Kim SD et al. World J Mens Health 2013;31: 53). Grayscale ultrasound (GSUS) reveals enlargement of the affected testicle and epididymis with a hypoechoic or heterogeneous appearance (Cokkinos DD et al. Insights Imaging 2013;4:185) with increased blood flow assessed by CDUS. Focal orchitis can promote abscess formation, a situation where the discrimination between these 2 entities is difficult, and sometimes 

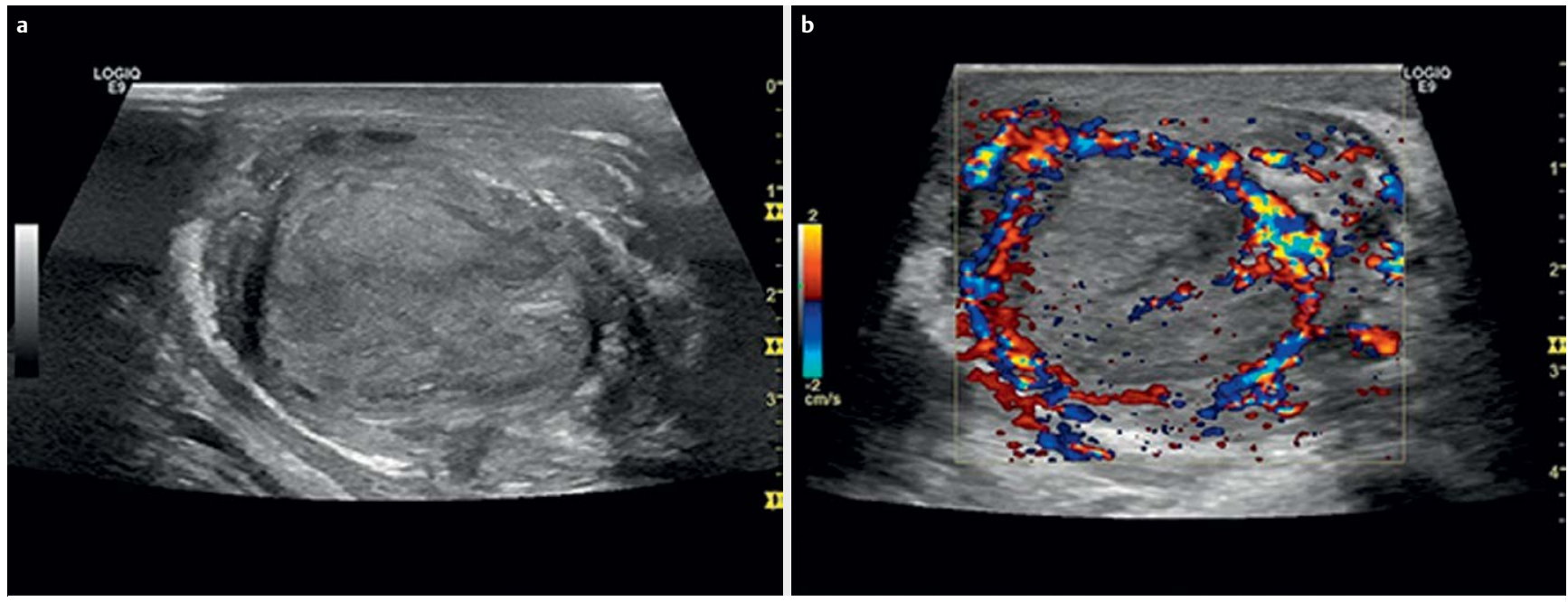

> Fig. 1 a Grayscale ultrasound of the left testicle showing inhomogeneous structure of the left testicle with thickened scrotal layers. b Color Doppler ultrasound showing marked epididymal congestion and only a few vascularized parenchymal areas of the testicle.
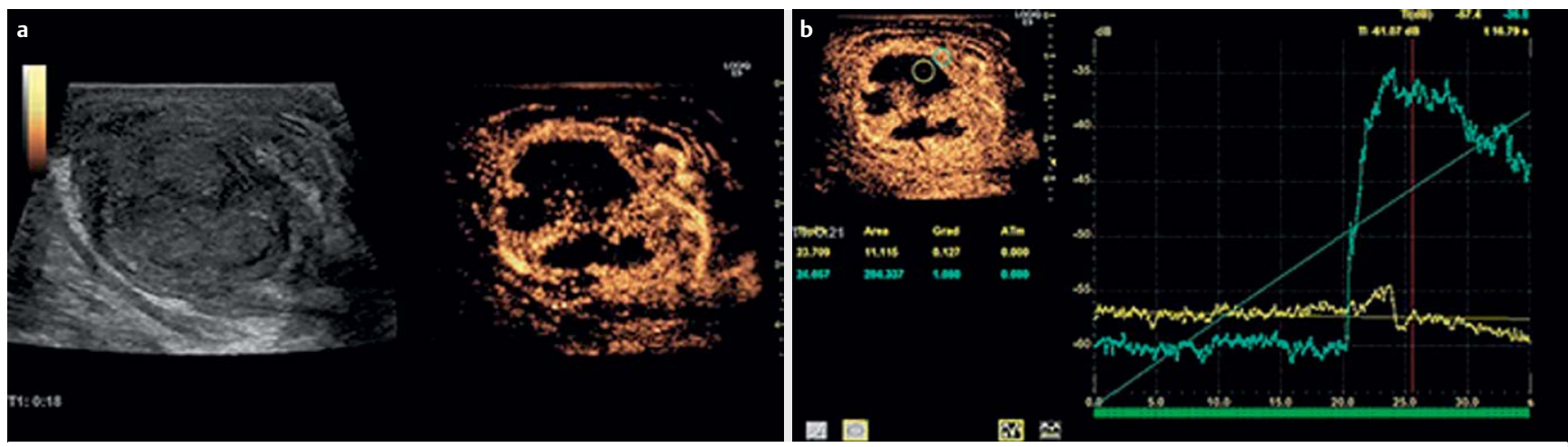

- Fig. 2 a Contrast-enhanced ultrasound of the left testicle showing no enhancement of the major part of the left testicle in the arterial phase. $\mathbf{b}$ Time-intensity curve analysis of contrast-enhanced ultrasound comparing 2 regions of interest (one of apparently normal, and one of altered testicular tissue) reveals an obvious difference of enhancement between these regions.
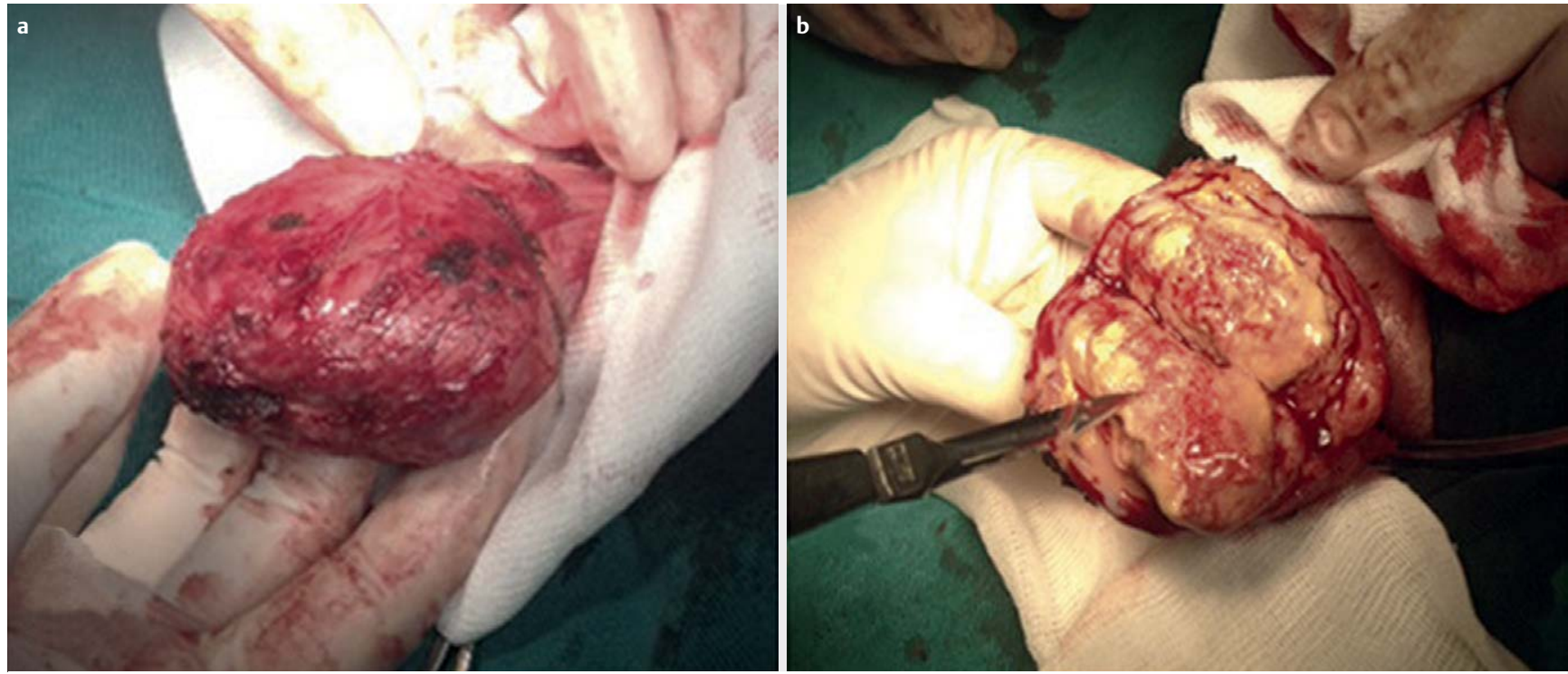

- Fig. 3 Intraoperatively the left testicle appears obviously damaged with a areas of necrosis on the surface and $\mathbf{b}$ areas of necrosis on the cut section as depicted by the scalpel tip. 

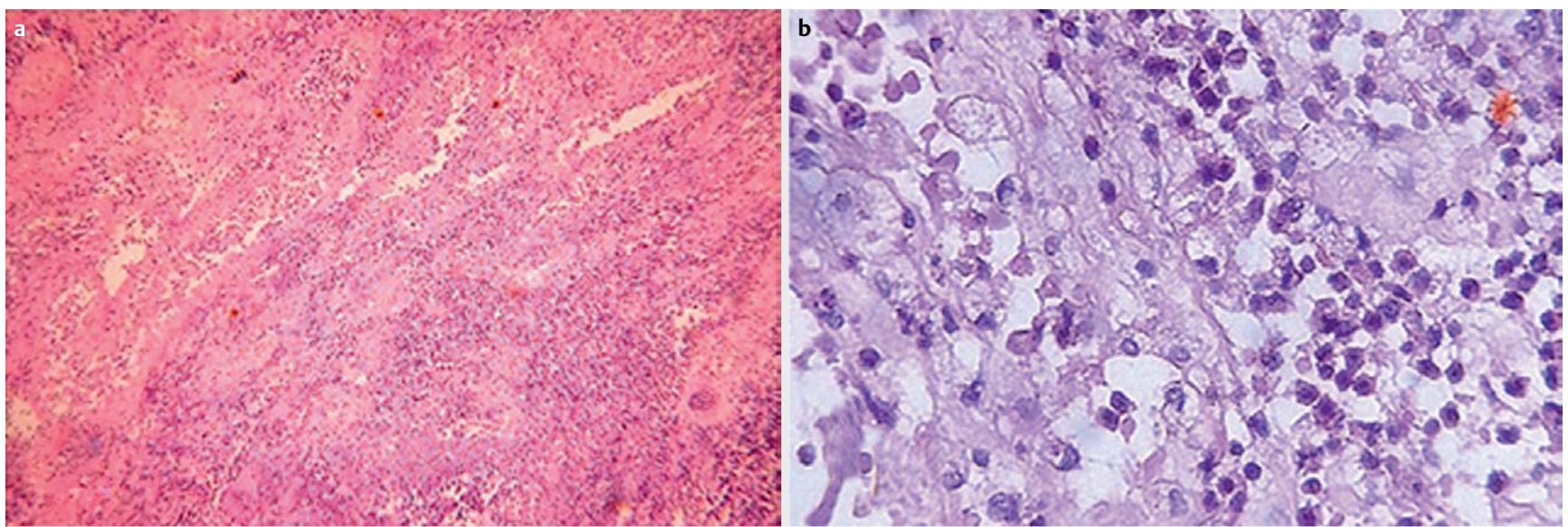

- Fig. 4 Histopathological assessment in hematoxylin-eosin staining showing testicle with a seminiferous tubules damaged by acute inflammation (magnification $4 \mathrm{x}$ ) and $\mathbf{b}$ polymorphonuclear neutrophil infiltration (magnification 40x).

requires differential diagnosis with tumors (Dudea SM et al. Med Ultrason 2010;12:43). CEUS reveals the inflamed tissue with hyperenhancement; no additional information is brought by CDUS. The real advantage of CEUS over GSUS and CDUS is identifying severe epididymo-orchitis, complicated by abscess formation (the unenhanced area surrounded by a hyperenhanced rim) even in the early stages or venous infarction (no enhancement) (Cokkinos DD et al. Insights Imaging 2013; $4: 185)$. CEUS is also helpful in differentiating epididymo-orchitis from testicular torsion, by revealing the absence of perfusion and assessing the extent of tissue damage (De Zordo T et al. Nat Rev Urol 2013;10:135).

In emergency situations, the first issue to be assessed is testicular perfusion, CDUS and PDUS being the methods of choice for this purpose, from the very beginning (Pavlica P et al. Eur Radiol 2001; 11: 22). CEUS, by using contrast agents that do not leave the vessels, evaluates blood flow better than CDUS and PDUS, since it is independent of movement and velocity of the flow (Wilson SR et al. Radiology 2010; 257: 24), even if the blood flow is stationary. Moreover, in emergency conditions, CEUS can be performed without having to wait for blood test results (renal function) since contrast agents are non-nephrotoxic, so that diagnosis is not delayed. Furthermore, US contrast agents are very well tolerated and have a lower risk of an allergic reaction (Wilson SR et al. Radiology 2010;257:24).

In patients with acute scrotal diseases, especially those with testicular torsion, who are difficult to handle in emergency departments due to their severe pain, CEUS might be challenging, but no more than the physical examination and conventional US and less than other imaging techniques. Since CEUS entails minimal discomfort for the patient, is non-irradiating and safe, takes only few minutes to be performed, and is available bedside without having to move the patient, it is most likely to be accepted by the patient. In addition to this, CEUS is more accurate in the final diagnosis than conventional GSUS and CDUS and has been proposed to be applied in emergency cases when US is inconclusive, without needing further imaging (Valentino $\mathrm{M}$ et al. Eur Radiol 2011; 21:1831).

Our case, as confirmed by the histology, was a case of acute epididymo-orchitis without remission of symptoms after longterm treatment with antibiotics, complicated by abscesses and necrosis of the testicle, as suggested by CEUS examination.

In conclusion, contrast-enhanced ultrasound is a low-risk, bedside examination method that is useful in acute scrotal and testicular diseases which could enable rapid diagnosis and the establishment of optimal treatment without the need for additional imaging techniques.
Authors

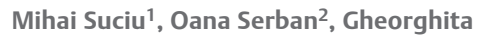
lacob $^{3}$, Ciprian Lucan ${ }^{4}$, Radu Badea ${ }^{5}$

1 Urology, Universitatea de Medicina si Farmacie Iuliu Hatieganu, Cluj-Napoca, Romania

2 Internal Medicine, Iuliu Hatieganu University of Medicine and Pharmacy, Cluj-Napoca, Romania

3 Pathology, Clinical Institute of Urology and Kidney Transplant, Cluj-Napoca, Romania

4 Urology, Iuliu Hatieganu University of Medicine and Pharmacy, Cluj-Napoca, Romania

5 Regional Institute of Gastroenterology and Hepatology, University of Medicine and Pharmacy, “Iuliu Hatieganu”, Cluj-Napoca, Cluj-Napoca, Romania

\section{Correspondence}

\author{
Dr. Oana Serban \\ Iuliu Hatieganu University of Medicine and \\ Pharmacy \\ Internal Medicine 8 \\ Victor Babes street \\ Cluj-Napoca \\ 400012 Romania \\ Tel.: + 40/264/597 256, \\ serban.oana@umfcluj.ro
}

Bibliografie

DOI http://dx.doi.org/10.1055/s-0042-122149

Published online: 2017 | Ultrasound Int Open

2017; 3: E46-E48

(c) Georg Thieme Verlag KG Stuttgart · New York ISSN 0722-1819 